\title{
(6) OPEN ACCESS \\ Progressive multifocal leucoencephalopathy in the rheumatic diseases: assessing the risks of biological immunosuppressive therapies
}

\author{
L H Calabrese, E S Molloy
}

Cleveland Clinic Lerner College of Medicine of Case Western Reserve University, Department of Rheumatic and Immunologic Diseases, Cleveland Clinic Foundation, Cleveland, Ohio, USA

Correspondence to:

Professor L H Calabrese,

Cleveland Clinic Lerner College of Medicine of Case Western

Reserve University, Department of Rheumatic and Immunologic Diseases, Cleveland Clinic

Foundation, 9500 Euclid Avenue, Cleveland, OH 44195, USA;

calabrl@ccf.org

Accepted 10 July 2008

\section{ABSTRACT}

Progressive multifocal leucoencephalopathy (PML) is a rare and often fatal opportunistic infection that has been well reported in patients with rheumatic diseases. The contributions of predisposing factors such as underlying disease and immunosuppressive drug selection are incompletely understood but it would appear that patients with systemic lupus erythematosus may be at highest risk. Natalizumab, a biological agent approved for multiple sclerosis and Crohn's disease has the clearest pattern of small but definite risk. Although the risk due to rituximab is difficult to assess given the multiple confounders, continued vigilance is warranted. Rheumatologists need to become familiar with PML and feel able to help patients make shared and informed decisions about the risks when starting treatment with immunosuppressive therapies. In particular, rheumatologists need to be vigilant and pursue the diagnosis of PML in all patients with unexplained neurological signs or symptoms with clinical and MRI findings compatible with the diagnosis.

Progressive multifocal leucoencephalopathy (PML) is a rare opportunistic infection of the central nervous system (CNS) encountered in immunosuppressed patients that until recently was primarily the concern of doctors caring for patients with HIV infection, cancer and organ transplants. A new era of interest and investigation of PML arrived when, during clinical trials of the immunomodulator natalizumab, an agent directed against $\alpha 4$ integrin approved for treatment of multiple sclerosis (MS), three patients (two with MS and one with Crohn's disease) developed PML. The drug was subsequently removed from the market, but later reintroduced for MS and more recently approved for Crohn's disease, but with significant restrictions and warnings. In December 2006 the US Food and Drug Administration issued a warning to healthcare providers about two reports of PML in rituximab-treated patients with systemic lupus erythematosus (SLE; an unapproved indication and outside of clinical trials). More recently (8 April 2008), the same agency issued an additional warning about reports of PML occurring in patients with a liver transplant treated with mycophenolate, an agent increasingly used in rheumatic disorders. Collectively, these data have given the rheumatology community pause and an impetus to reflect on this rare complication, including its basic biology, diagnosis and treatment and the risks it may pose to patients. This subject has recently been the topic of several reviews. ${ }^{12}$

\section{PML: THE DISEASE}

The aetiological agent of PML is the JC virus, named for the initials of the patient from whom it was initially isolated. JC is a member of the polyomavirus genus of the Papovaviridae family. The pathogenesis of PML is incompletely understood but appears to begin with infection early in childhood, presumably via an oral-pharyngeal route, establishing a lifelong latency with $>80 \%$ of adults harbouring specific antibodies. In noncompromised hosts the virus is intermittently shed in the urine without clinical sequelae. A number of critical steps are required to convert from this state of asymptomatic latent infection to the fully developed disease state. These include: (a) establishment of latent infection in extraneural tissues (especially the kidney but also bone marrow, spleen, tonsils and other tissues); (b) critical rearrangements within the regulatory regions of the virion which convert it from its archetype to a neurotrophic form; (c) reactivation of viral infection with resultant viraemia and entry into the CNS; (d) failure of immunological control; (e) infection of myelin-producing cells in the brain (ie, oligodendrocytes) with resultant disease. ${ }^{3}$

\section{CLINICAL AND LABORATORY FEATURES OF PML}

The most common presentation of PML is that of a subacute focal neurological disorder with motor weakness, visual loss and lack of coordination combined with higher cortical impairment, including confusion, disorientation and personality changes. Virtually all patients with PML exhibit MRI changes, most frequently asymmetrical, subcortical diffuse changes in white matter that are hyperintense on T2 and FLAIR sequences and hypointense on T1-weighted images. ${ }^{4}$ Such lesions typically do not enhance or suggest oedema but exceptions do occur. ${ }^{5}$

As there are no neurological symptoms or signs pathognomonic for PML, this disease can be difficult to distinguish from MS and other neuroinflammatory conditions such as neuropsychiatric SLE and CNS vasculitis. Helpful clues include the facts that PML does not present ictally, evolves progressively over weeks and spares the spinal cord and optic nerve. The diagnosis of PML is made by identifying JC virus in the cerebral spinal fluid by PCR, which has a test sensitivity of about $70-80 \%$ and a specificity approaching $100 \%{ }^{3}$ Identifying the JC virus in urine or blood has no diagnostic value. The cerebral spinal fluid itself is generally remarkably bland being frequently normal or alternatively, displaying a mild pleocytosis and raised protein level. 
PML is often fatal and if patients do survive it is often with significant sequelae. There are no known effective treatments and all efforts should be directed at minimising or reducing immunosuppressive therapies when possible.

\section{EPIDEMIOLOGY OF PML IN RHEUMATIC DISEASES}

We previously reported a review of 36 cases of PML occurring in patients with rheumatic diseases. ${ }^{1}$ We excluded cases where there was insufficient information to substantiate the diagnoses of rheumatic disease or PML, or both. Since that time, an additional five proven cases of PML in patients with rheumatic diseases have been reported in the English language medical literature, ${ }^{6-10}$ including one of two cases of PML in rituximabtreated patients with SLE, ${ }^{7}$ initially described in the alert released by the US Food and Drug Administration in 2006. ${ }^{11}$ In addition, four cases of PML developing in patients with rheumatic diseases have been reported in abstract form. ${ }^{12}$ Overall, of the additional 10 cases mentioned above, seven patients had SLE. ${ }^{6911} 12$ Of the other three patients, one had rheumatoid arthritis, ${ }^{12}$ one had a 3-year history of destructive polyarthritis and Raynaud's phenomenon with positive antinuclear antibodies and Jo-1 antibodies and CD4+ lymphopenia ${ }^{10}$ and one patient with Sjögren's syndrome developed PML and CD4+ lymphopenia. ${ }^{8}$ A striking observation was that about $40 \%$ of the patients with SLE (and 25\% of the patients without SLE) who developed PML had been treated with minimal immunosuppressive therapy in the 6 months before the diagnosis of PML, and only $33 \%$ and $62 \%$ of the SLE and non-SLE groups, respectively, had ever been treated with alkylator therapy. ${ }^{1}$ These findings suggest that the risk of PML in patients with rheumatic diseases and in patients with SLE, in particular, is not wholly attributable to the intensity of iatrogenic immunosuppression. The mechanism for any posited predisposition to the development of PML in such patients is currently unknown.

\section{WORKING HYPOTHESIS ON THE EFFECTS OF DISEASE, BIO- LOGICAL AND NON-BIOLOGICAL IMIMUNOSUPPRESSIVE AGENTS}

At the present time, while our overall understanding of PML is plagued by considerable uncertainty, a few conclusions can be cautiously put forth. First, it appears that SLE is a special case since about two-thirds of all cases of PML reported in rheumatic diseases have been in patients with SLE. Even more telling is the fact that so many patients with SLE-PML have histories that reflect modest levels of immunosuppression, suggesting that SLE itself may be a predisposing factor, as discussed above. Second, a crude hierarchy of the risks of biological therapeutic agents can be proposed from the available data (table 1).

Clearly, natalizumab holds a special level of risk, given that incident cases have been reported in clinical trials, supporting an incidence of about 1 per 1000 per 18-month observation period. Although no further cases have been reported, even after exhaustive case-finding efforts, this agent stands alone in its defined epidemiological risk.

Table 1 Summary of the risk of multifocal leucoencephalopathy associated with biological therapies

\begin{tabular}{lll}
\hline Agent & Level of evidence & Basis for evidence \\
\hline Natalizumab & Strong & Rare definite cases in clinical trials \\
Rituximab & Uncertain & Rare definite cases outside clinical trials \\
TNF inhibitors & Uncertain* & No definite cases \\
Abatacept & None & -
\end{tabular}

\footnotetext{
*This may be under-reported. ${ }^{13}$
}

TNF, tumour necrosis factor.
Rituximab has also been associated with PML in both oncological and rheumatic diseases but in this situation its absolute risk is problematic. Confounders include the fact that none of the cases reported have been in clinical trials, multiple other immunosuppressive drugs have been used (eg, purine analogues, stem cell transplantation regimens), especially in oncological disease, and no cases have yet been described in patients with rheumatoid arthritis, but rather in SLE, which may be associated with a predisposition to PML, as noted above.

In published reports of tumour necrosis factor (TNF) inhibitor, although "demyelinating disease" is listed as a potential adverse event, no documented (ie, JC PCR positive or brain biopsy proven) cases of PML have been reported. For these agents a lingering concern is the possibility that atypical and possibly self-limited or abortive forms of PML might have occurred given that all approved agents have been associated with unexplained white matter lesions of which only a few have been biopsied, leaving unanswered the pathological basis for most. ${ }^{13} 14$

No data are available to allow an assessment of the risk of other agents, but this does not mean that there is no risk. As PML is a rare disease, clinical trials of biological therapeutic agents are too small to rule out such effects. Thus postmarketing surveillance is critical.

Quantifying risks of other forms of (non-biological) immunosuppressive drugs in the treatment of rheumatic diseases is difficult given the rarity of reported cases. The recent FDA warning on mycophenolate use after liver transplantation is of concern given the increasing used of this non-approved drug in the treatment of SLE.

Funding: Both authors are supported by the $\mathrm{R} \mathrm{J}$ Fasenmyer Center for Clinical Immunology.

Competing interests: None.

\section{REFERENCES}

1. Calabrese LH, Molloy ES, Huang D, Ransohoff RM. Progressive multifocal leukoencephalopathy in rheumatic diseases: evolving clinical and pathologic patterns of disease. Arthritis Rheum 2007:56:2116-28.

2. Boren EJ, Cheema GS, Naguwa SM, Ansari AA, Gershwin ME. The emergence of progressive multifocal leukoencephalopathy (PML) in rheumatic diseases. $J$ Autoimmun 2008;30:90-8.

3. Berger JR. Progressive multifocal leukoencephalopathy. Current Neurol Neurosci Rep 2007:7:461-9.

4. Kuker W, Mader I, Nagele T, Uhl M, Adolph C, Klose U, et al. Progressive multifocal leukoencephalopathy: value of diffusion-weighted and contrast-enhanced magnetic resonance imaging for diagnosis and treatment control. Eur J Neurol 2006;13:819-26.

5. Huang D, Cossoy M, Li M, Choi D, Taege A, Staugaitis SM, et al. Inflammatory progressive multifocal leukoencephalopathy in human immunodeficiency virusnegative patients. Ann Neurol 2007;62:34-9.

6. Govindappa V, Hicks S, Wichter M, Jolly M. Progressive multifocal leukoencephalopathy in systemic lupus erythematosus. Arthritis Rheum 2007:57:352-4.

7. Harris HE. Progressive multifocal leucoencephalopathy in a patient with systemic lupus erythematosus treated with rituximab. Rheumatology (Oxford) 2008; 47:224-5

8. Hayashi Y, Kimura A, Kato S, Koumura A, Sakurai T, Tanaka Y, et al. Progressive multifocal leukoencephalopathy and CD4+ T-lymphocytopenia in a patient with Sjögren syndrome. J Neurol Sci 2008;268:195-8.

9. Roberts JR, Finger DR. Progressive multifocal leukoencephalopathy mimicking central nervous system lupus. J Rheumatol 2007;34:2119-20.

10. Viallard JF, Lazaro E, Ellie E, Eimer S, Camou F, Caubet 0 , et al. Improvement of progressive multifocal leukoencephalopathy after cidofovir therapy in a patient with a destructive polyarthritis. Infection 2007;35:33-6.

11. Anonymous. Rituxan warning. FDA consumer 2007;41:3.

12. Jonsen A, Bengtsson AA, Sturfelt G, Nived O. A difficult diagnosis: four cases of progressive multifocal leukoencephalopathy over two decades in one rheumatological unit [abstract]. Arthritis Rheum 2007;56:S461.

13. Roos JC, Ostor AJ. Neurological complications of infliximab. J Rheumatol 2007;34:236-7; author reply 7-8.

14. Mohan N, Edwards ET, Cupps TR, Oliverio PJ, Sandberg G, Crayton H, et al. Demyelination occurring during anti-tumor necrosis factor alpha therapy for inflammatory arthritides. Arthritis Rheum 2001;44:2862-9. 